\section{Obinutuzumab in connective tissue diseases after former rituximab-non-response: a case series}

In the following case series, we present four patients with different connective tissue diseases (CTD) showing a remarkably positive response on treatment with Obinutuzumab despite former rituximab-non-response in three cases. Demographic data including, age, gender, disease duration, type of involvement, previous as well as concomitant treatments are shown in table 1. Efficacy of treatment was assessed by clinical, laboratory and radiologic findings or global patient assessment for rheumatological symptoms, respectively. Clinical response was defined by an improvement of involved organ functions as well as a reduction of the severity of symptoms. Global tolerance was evaluated.

SLE

Two patients with SLE and active glomerulonephritis were treated with Obinutuzumab after rituximab failure. One patient each additionally suffered from antiphospholipid syndrome and neuropsychiatric lupus, respectively. After one cycle with obinutuzumab (1 g, day 0,14$)$, both patients came off dialysis and showed a stable kidney function over a time period of at least 6 months. One patient had cardiac involvement and highly elevated NT-pro-BNP which markedly decreased after treatment with obinutuzumab. Serological markers such as anti-ds-DNA antibodies and C3-complement consumption strongly improved after therapy.

\section{ANTI-J01 SYNDROME}

We further included a patient with anti-Jo1-syndrome who did not respond to her previous treatments including Rituximab, IVIG, Cyclophosphamide and repeated prednisolone pulse therapies. Her disease was manifested by myositis (creatinekinase (CK) $\max .8946 \mathrm{U} / \mathrm{L}$ ) and CT-confirmed interstitial lung disease with a decreased CO-diffusion capacity of $57.3 \%$ expected. After one cycle of obinutuzumab, muscle weakness improved and CK and lactate dehydrogenase levels markedly decreased.

\section{CREST SYNDROME}

In this patient, CREST syndrome was diagnosed with sclerodactyly, Raynaud's phenomenon, oesophageal hypomotility, teleangiectasia, calcinosis cutis and pulmonary arterial hypertension and an ANA-titre of 1:10000 in 2006. In 2013, she developed chronic lymphocytic leukaemia requiring a $\mathrm{B}$-cell depleting treatment for which obinutuzumab was chosen in accordance with current national and European guidelines.

After two cycles of obinutuzumab, the patient had a complete remission of the haematological disease and showed diminishing calcinosis cutis which gradually disappeared completely until the end of the treatment.

Table 1 Patient demographics and history of diseases and treatments

\begin{tabular}{|c|c|c|c|c|}
\hline & SLE 1 & SLE 2 & $\begin{array}{l}\text { Anti-Jo1 } \\
\text { syndrome }\end{array}$ & CREST-syndrome/CLL \\
\hline Age & 33 & 52 & 46 & 80 \\
\hline Gender & $\mathrm{F}$ & $\mathrm{F}$ & $\mathrm{F}$ & $\mathrm{F}$ \\
\hline Year of diagnosis & 2019 & 2008 & 2019 & $\begin{array}{l}2006 / \\
2013\end{array}$ \\
\hline Clinical manifestations & $\begin{array}{l}\text { Nephritis, polyserositis, } \\
\text { pancytopaenia, pancarditis }\end{array}$ & $\begin{array}{l}\text { Nephritis, CNS-involvement, Libman- } \\
\text { Sacks endocarditis, APS, ILD }\end{array}$ & Myositis, ILD & $\begin{array}{l}\text { Sclerodactylia, teleangiectasia, } \\
\text { Raynaud's, PAH, calcinosis cutis }\end{array}$ \\
\hline $\begin{array}{l}\text { Previous therapies and dosage } \\
\text { of prednisolone before treatment } \\
\text { with } \mathrm{OBI}\end{array}$ & $\begin{array}{l}\text { CYC } 6 \times 500 \text { mg i.v., MPA, RTX } \\
2 \times 1 \mathrm{~g} \text { i.v. twice within } 6 \text { months; } \\
\text { prednisolone } 80 \mathrm{mg} / \mathrm{d}\end{array}$ & $\begin{array}{l}\text { CYC } 6 \times 500 \text { mg i.v., MPA, RTX } 2 \times 1 \mathrm{~g} \\
\text { i.v. twice within } 6 \text { months, IVIG, } \\
\text { plasmapheresis; prednisolone } 70 \mathrm{mg} / \mathrm{d}\end{array}$ & $\begin{array}{l}\text { CYC } 6 \times 750 \mathrm{mg} \text { i.v., RTX } \\
2 \times 1 \mathrm{~g} \text { i.v., IVIG; repeated } \\
\text { prednisolone pulse therapies } \\
\text { starting with } 80 \mathrm{mg} / \mathrm{d}\end{array}$ & SSZ, MTX, HCQ \\
\hline $\begin{array}{l}\text { Characteristic findings before } \\
\text { treatment with Obinutuzumab }\end{array}$ & $\begin{array}{l}\text { Crea 3,14 mg/dL, dialysis, } \\
\text { erythrocyturia } 3327 / \mu \mathrm{L} \text {, anti-ds- } \\
\text { DNA ab. (RIA) 15128,6IU/mL, C3 } \\
\text { 0,28 g/L, anti-nucleos. ab. 130,9 IE/ } \\
\text { mL, nt-pro BNP } 42526 \mathrm{ng} / \mathrm{L} \text {, } \\
\text { SLEDAI-2K: } 30\end{array}$ & $\begin{array}{l}\text { Crea } 4,97 \mathrm{mg} / \mathrm{dL} \text {, dialysis, Prot. Urine/ } \\
\text { Crea Urine } 108,27 \mathrm{~g} / \mathrm{mol} / \mathrm{Kr} \text {, anti-ds- } \\
\text { DNA ab. (RIA) 2,5IU/mL, } \\
\text { SLEDAI-2K: } 32\end{array}$ & $\begin{array}{l}\text { myalgia, muscle weakness, } \\
\text { dyspnoea, CK } 8946 \text { U/L, LDH } \\
910 \text { U/L, CRP 13,9 mg/L }\end{array}$ & $\begin{array}{l}\text { calcinosis cutis, PA } \\
65 / 30 / 43 \mathrm{mmHg}, \mathrm{PC} 13 \mathrm{mmHg}, \\
\text { PAR } 393 \text { dyn.sec.cm }-5, \mathrm{Cl} 3,3 \mathrm{l} / \\
\mathrm{min}^{*} \mathrm{~m}^{2}, \mathrm{VC} \text { in } 75,6 \%, \mathrm{FEV} 1 \\
71,0 \%, \mathrm{FVC} 81,2 \%, \mathrm{TLC} 85,6 \% \text {, } \\
\text { Rtot } 96,4 \%, \mathrm{DLCOC} \mathrm{SB} 49,7 \% \text {, } \\
\text { DLCOc/VA 73,6\%" }\end{array}$ \\
\hline $\begin{array}{l}\text { Characteristic findings after } \\
\text { treatment with Obinutuzumab }\end{array}$ & $\begin{array}{l}\text { Crea } 1,3 \mathrm{mg} / \mathrm{dL} \text {, no dialysis, } \\
\text { erythrocyturia } 258 / \mu \mathrm{L} \text {, anti-ds-DNA } \\
\text { ab. (RIA) } 224,9 \mathrm{IU} / \mathrm{mL}, \mathrm{C} 30,83 \mathrm{~g} / \mathrm{L} \text {, } \\
\text { anti-nucleos. ab. 20,2 IE/mL, nt-pro } \\
\text { BNP } 520 \mathrm{ng} / \mathrm{L} \text {, } \\
\text { SLEDAI-2K: } 2\end{array}$ & $\begin{array}{l}\text { Crea } 1,95 \mathrm{mg} / \mathrm{dL} \text {, no dialysis, Prot. } \\
\text { Urine/Crea Urine 57,16 g/molKr, anti- } \\
\text { ds-DNA ab. (RIA) 2,5 IU/mL, } \\
\text { SLEDAI-2K: } 17\end{array}$ & $\begin{array}{l}\text { myalgia and muscle weakness } \\
\text { strongly diminished, no } \\
\text { dyspnoea, CK } 188 \mathrm{U} / \mathrm{L} \text {, LDH } \\
221 \mathrm{U} / \mathrm{L} \text {, CRP } 4,1 \mathrm{mg} / \mathrm{L}\end{array}$ & $\begin{array}{l}\text { calcinosis cutis disappeared, VC } \\
\text { in } 85,5 \% \%, \text { FEV1 } 82,9 \% \text {, FVC } \\
90,7 \% \text {, TLC 93,5\%, Rtot } 84 \% \text {, } \\
\text { DLCOc SB 49,3\%, DLCOc/VA } \\
69,2 \%\end{array}$ \\
\hline $\begin{array}{l}\text { Co-medication during treatment } \\
\text { with } \mathrm{OBI} \text { and dosage of } \\
\text { prednisolone after treatment with } \\
\mathrm{OBI} \text { at last follow-up }\end{array}$ & $\begin{array}{l}\text { MPA } 360 \mathrm{mg} 2-0-2, \text { HCQ } 200 \mathrm{mg} \\
1-0-0, \text { prednisolone } 3 \mathrm{mg} 1-0-0\end{array}$ & $\begin{array}{l}\text { MPA } 360 \text { mg 2-0-2, HCQ } 200 \text { mg 1-0-0, } \\
\text { prednisolone } 5 \text { mg 1-0-0 }\end{array}$ & $\begin{array}{l}\text { AZA } 50 \mathrm{mg} 1-1 / 2-1, \\
\text { prednisolone } 5 \mathrm{mg} 1-0-0\end{array}$ & $\begin{array}{l}\text { macitentane } 10 \mathrm{mg} 1-0-0 \\
\text { chlorambucile, bendamustine }\end{array}$ \\
\hline Global tolerance & No major side effects & No major side effects & No major side effects & No major side effects \\
\hline
\end{tabular}




\section{CONCLUSION AND PHARMACOLOGICAL CONSIDERATIONS}

Obinutuzumab has recently been proven as an effective option in proliferative lupus nephritis leading to significantly better renal response compared with placebo. ${ }^{1}$ The data presented here suggest an efficacy of obinutuzumab in different CTD even after failure of rituximab. We hypothesise that the low dependency of complement factors, the altered mechanisms of action including enhanced antibody-dependent cellular cytotoxicity (ADCC) of obinutuzumab and its presumably enhanced efficacy in inflamed tissues are factors supporting our hypothesis that obinutuzumab should be studied in various CTD after rituximab failure, but especially as first-line biologic after failure of conventional disease-modifying antirheumatic drugs (DMARDs). ${ }^{2-5}$

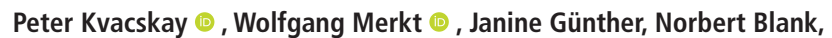 Hanns-Martin Lorenz}

Department of Internal Medicine V Hematology Oncology Rheumatology, University Hospital Heidelberg, Heidelberg, Germany

Correspondence to Dr Peter Kvacskay, Department of Internal Medicine V Hematology Oncology Rheumatology, University Hospital Heidelberg, Heidelberg 69120, Germany; peter.kvacskay@med.uni-heidelberg.de

\section{Handling editor Josef S Smolen}

Contributors We ensure that the given number and order of authors was accepted by all participants and that all authors listed were actively taking part on collecting and processing data as well as on writing and reviewing the given manuscript.

Funding The study was supported by: Medical Faculty of University of Heidelberg, Eva Luise and Horst Köhler Foundation (to WM).

Competing interests WM, NB and $\mathrm{H}-\mathrm{ML}$ have received consulting fees, speaking fees and/or honoraria from Roche (less than US\$10 000 each), and unallocated funds for research from Roche ( $€ 15000)$

Patient and public involvement Patients and/or the public were not involved in the design, or conduct, or reporting, or dissemination plans of this research.

Patient consent for publication Not applicable.

Provenance and peer review Not commissioned; externally peer reviewed.

\section{(2) \\ OPEN ACCESS}

Open access This is an open access article distributed in accordance with the Creative Commons Attribution Non Commercial (CC BY-NC 4.0) license, which permits others to distribute, remix, adapt, build upon this work non-commercially, and license their derivative works on different terms, provided the original work is properly cited, appropriate credit is given, any changes made indicated, and the use is non-commercial. See: http://creativecommons.org/licenses/by-nc/4.0/.

(C) Author(s) (or their employer(s)) 2022. Re-use permitted under CC BY-NC. No commercial re-use. See rights and permissions. Published by BMJ.

\section{Check for updates}

To cite Kvacskay P, Merkt W, Günther J, et al. Ann Rheum Dis Epub ahead of print: [please include Day Month Year]. doi:10.1136/annrheumdis-2021-221756

Received 3 November 2021

Accepted 5 January 2022

Ann Rheum Dis 2022;0:1-2. doi:10.1136/annrheumdis-2021-221756

ORCID iDs

Peter Kvacskay http://orcid.org/0000-0003-2925-4066

Wolfgang Merkt http://orcid.org/0000-0002-3108-154X

\section{REFERENCES}

1 Furie RA, Aroca G, Cascino MD, et al. B-Cell depletion with obinutuzumab for the treatment of proliferative lupus nephritis: a randomised, double-blind, placebocontrolled trial. Ann Rheum Dis 2022:81:100-7.

2 Mössner E, Brünker P, Moser S, et al. Increasing the efficacy of CD20 antibody therapy through the engineering of a new type II anti-CD20 antibody with enhanced direct and immune effector cell-mediated B-cell cytotoxicity. Blood 2010;115:4393-402.

3 Tobinai K, Klein C, Oya N, et al. A review of Obinutuzumab (GA101), a novel type II anti-CD20 monoclonal antibody, for the treatment of patients with B-cell malignancies. Adv Ther 2017;34:324-56.

4 Reddy V, Klein C, Isenberg DA, et al. Obinutuzumab induces superior B-cell cytotoxicity to rituximab in rheumatoid arthritis and systemic lupus erythematosus patient samples. Rheumatology 2017:56:1227-37.

5 Marinov AD, Wang H, Bastacky SI, et al. The type II anti-CD20 antibody Obinutuzumab (GA101) is more effective than rituximab at depleting B cells and treating disease in a murine lupus model. Arthritis Rheumatol 2021;73:826-36. 